\title{
Salaires des assistantes médicales pour 2004 et recommandations de salaires
}

\author{
Dr Michel Marchev ${ }^{a}$, Dr Jürg Kremo ${ }^{b}$, Elwina Kaufmann ${ }^{c}$, Me Bruno Gutknecht ${ }^{d}$
}

Etant donné que, depuis 1991, les sociétés cantonales de médecine élaborent leurs propres recommandations de salaires pour les assistantes médicales, nous renonçons désormais à publier des recommandations salariales pour l'ensemble de la Suisse.

Les conditions-cadres ci-après conservent toutefois leur valeur pour fixer individuellement le salaire d'une assistante médicale.

\section{Les conditions-cadres}

\section{Formation}

- Diplôme de la Fédération des médecins suisses (estampille) resp. certificat de capacité de la FMH avec certificat de radioprotection et autorisation de faire des radiographies;

- Certificat fédéral de capacité d'assistante médicale.

\section{Conditions de travail: bases de calcul}

- Semaine de 43 heures en moyenne annuelle;

- 4 semaines de vacances (assistantes médicales de moins de 20 ans et de plus de 50 ans: 5 semaines).

Toute dérogation importante à ces conditions sera répercutée sur le salaire.

\section{Normes régionales}

Les salaires doivent être adaptés aux conditions régionales.

\section{$13^{\mathrm{e}}$ salaire}

Un $13^{\text {e }}$ salaire doit être versé à l'assistante médicale à la fin de l'année. Si les rapports de service n'ont pas duré pendant toute l'année civile, le $13^{\text {e }}$ salaire sera versé au prorata des mois d'engagement.

\section{Prime d'ancienneté et augmentation du salaire réel}

Le montant de la prime d'ancienneté et d'une éventuelle augmentation du salaire réel est fixé au cours de l'entretien annuel sur les qualifications de l'employée.

\section{Compensation du renchérissement}

Les parties conviennent à la fin de chaque année de l'octroi d'une compensation du renchérissement et, dans l'affirmative, de son envergure (ISPC septembre: 0,5\%; octobre: 0,5\%).

Lorsque la compensation du renchérissement est convenue par contrat, elle doit être accordée.

\section{Travail à temps partiel rétribué au mois}

Pour le travail à temps partiel, le salaire brut recommandé s'élève au $1 / 43$ du salaire brut entier multiplié par le nombre des heures de travail hebdomadaire convenu (base de calcul: 43 heures hebdomadaires).

\section{Salaire horaire}

Un salaire horaire est recommandé en cas d'horaire de travail très réduit et en même temps irrégulier. Le taux horaire conseillé est de $6 \%$ du salaire mensuel (le $13^{\mathrm{e}}$ salaire y étant inclus au pro rata). Il sera complété par une contribution aux vacances de 8,33\% correspondant aux quatre semaines de vacances habituelles (pour 5 semaines le taux sera de 10,64\% et pour 6 semaines $13,04 \%$ etc.). Ce mode de calcul est également valable pour le paiement des heures supplémentaires.

\section{Heures supplémentaires}

La réglementation des heures supplémentaires a toujours fait l'objet de demandes de renseignements. Lors de la révision du contrat de travail au début de l'année 2001, ce point a été clarifié. Au chiffre 2 du contrat, il est désormais possible d'opter pour les solutions suivantes:

- La compensation des heures supplémentaires par du temps libre ou des vacances d'une même durée dont la date est décidée par l'employeur.

- L'indemnisation des heures supplémentaires par une majoration de salaire de $25 \%$. Le taux horaire est de $6 \%$, d'un plein salaire mensuel, auquel s'ajoute la contribution aux vacances (cf. «salaire horaire»). d Secrétaire central, Association suisse des assistantes médicales SVA 
Les dispositions et recommandations suivantes s'appliquent à tous les salaires:

Déductions sur le salaire brut

- AVS, AI, APG, ACI: 6,3\% (AVS, AI, APG = $5,05 \%, \mathrm{ACI}=1,25 \%$ )

- Assurance-accidents non professionnels: $1,367 \%$ (pour un engagement de plus de 8 heures par semaine)

- Prévoyance professionnelle (2 $2^{\mathrm{e}}$ pilier LPP): part de l'employée à la contribution (habituellement $50 \%$ ) calculée en fonction de l'âge, selon le certificat d'assurance.

\section{Contrats de travail et autres conditions} d'engagement

Nous préconisons d'établir le contrat par écrit, sur la formule de la FMH créée conjointement avec les associations des assistantes médicales, accompagnée des recommandations cantonales. Ces documents peuvent être obtenus aux adresses suivantes:

- Fédération des médecins suisses FMH, Elfenstrasse 18, 3000 Berne 16, tél. 03135911 11, www.fmh.ch, e-mail: fmhmprax@hin.ch;

- Association romande des assistantes médicales ARAM, case postale 2034, 1002 Lausanne, tél. 02194345 64;
- Association suisse des assistantes médicales SVA, case postale 6432, 3001 Berne, tél. 031 3811443 www.sva.ch, e-mail: sekretariat @sva.ch;

- Berufsverband Medizinischer Praxisassistentinnen BMPA, Sonnenbergstrasse 7, 6005 Luzern, tél. 0413102223 , www.bsmpa.ch, e-mail: bsmpa@bluewin.ch;

- Fédération suisse des associations d'assistantes médicales FSAAM, chemin du Lussy 18, 1806 St-Légier, tél. 02194345 64, www.fsaam.ch, e-mail: fsaam-ch@bluewin. ch.

L'Association d'assistantes médicales du canton de Genève a une convention collective de travail et l'Association du Tessin a son propre modèle de contrat de travail. Les deux sont élaborés avec les sociétés cantonales de médecine respectives et disponibles aux adresses suivantes:

- Association genevoise des assistantes médicales AGAM, rue des Pavillons 11, 1205 Genève, tél. 02232110 69;

- Associazione ticinese assistenti di studio medico ATAM, Via Ronchetto, 6814 Cadempino, tel. 0919947835.

Les salaires des apprenties se basent sur les recommandations cantonales. 